\title{
A Short Review on 3-d Printing of FRP Composites Using Stereolithography
}

\author{
Girish Dutt Gautam*, Sunita Rani**, Sudhanshu Raghuwanshi***, Samendra Singh****, Shweta Choudhary***** \\ and Jyotsna Pandit******

\begin{abstract}
*Department of Mechanical Engineering, Mangalmay Institute of Engineering and Technology, Greater Noida, UP (India)
**Department of Information Technology, Jagannath International Management School, New Delhi (India)

***Department of Information Technology, Shark Tech Intelligence Pvt. Ltd., Noida, UP (India)

****Department of Computer Science \& Engineering, Mangalmay Institute of Engineering and Technology, Greater Noida, UP (India)

*****Department of Applied Sciences \& Humanities, ABES Engineering College, Ghaziabad, UP (India)

******Department of Applied Sciences \& Humanities, Mangalmay Institute of Engineering and Technology, Greater Noida, UP (India)

Corresponding Author : girish.gautam1985@gmail.com
\end{abstract}

\begin{abstract}
A higher product diversification range with excellent physical, mechanical and chemical properties make Fiber-reinforced polymer (FRP) composite materials a prominent candidate for engineering applications. But, conventional manufacturing techniques always face critical issues during the development of FRP's complex and intrinsic profile. In recent years, Additive Manufacturing (AM) or 3-D printing proves itself a robust technique to produce application-specific parts of FRP composites with a higher degree of customization. In comparison to other 3D printing techniques, Stereolithography (SLA) is able to create mechanically stable objects with higher processing speed. This information paves the way for the present review article. This paper reviews the recent advancement of SLA technique to develop objects of FRP composite materials.
\end{abstract}

Ke y w ord s : 3D printing; fiber; Polymer composite; Stereolithography.

\section{INTRODUCTION}

In recent decades, 3D Printing or additive manufacturing (AM) technique is emerged as a robust rapid tooling and manufacturing technique to produce parts having complex geometries with reduced volume of the waste material (Frketic, Dickens, \& Ramakrishnan, 2017). Higher versatility at low cost of 3D printing technique is continuously increasing its applications in the automotive, biomedical, aerospace, design etc. industries. Its higher customizability makes it suitable to fabricate complex structures and intrinsic profiles (Kothman \& Faber, 2016; Noorani, 2017; Yan et al., 2018).

In 1986, Charles Hull demonstrated this technique as a material joining process in successive layers to make objects by using data of its 3D model. This technique consists four phases such as 3D CAD modelling, STL file creation, slicing and printing. In the initial phase, a CAD software is employed to prepare a meshed 3D model of the required object. Then, a STL (Surface Tessellation Language) file is developed for the prepared 3D model. In the next phase, mesh data of the model is sliced into the 2D layers. Thereafter, this $2 \mathrm{D}$ sliced data is sent to the suitable machine for 3D printing of the object (Hull, 2015; Wong \& Hernandez, 2012).

In industries, 3D printing technique is majorly using for printing of single material objects. Thereby, improvements in the physical and chemical properties of printed parts are limited. This is a very serious issue which limits the industrial applications of 3D printing. To overcome this issue, various researchers have focused on multi-material printers to fabricate composite materials. These printers are also partially able to control the properties and compositions 
of the using material. In recent year, 3D printing is gaining intense attention of researchers for fabricating fiber reinforced composite (FRC) objects.

$3 \mathrm{D}$ printing has various techniques to fabricate the object. Cost and speed of the process, desired specific properties, appearance and geometry of fabricated object are some of the vital criteria which are responsible to select a process type. SLA, laminated object manufacturing (LOM), fused deposition modeling (FDM), selective laser sintering (SLS), and extrusion are the major techniques for additive manufacturing of FRC materials (Dickson, Barry, McDonnell, \& Dowling, 2017; Mohammadizadeh, Imeri, Fidan, \& Elkelany, 2019; Ran et al., 2021; Yavas, Zhang, Liu, \& Wu, 2021). However, in these techniques, fiber orientation, void contents, matrix cracking are the key issues to be addressed. In 3D printing of polymers, filler materials like nanoparticles, CNT's, graphene can be blended to the base material to improve their mechanical, electrical and physical properties (Bose, Ke, Sahasrabudhe, \& Bandyopadhyay, 2018; Stavropoulos \& Foteinopoulos, 2018; Zhou, Fu, \& He, 2020). This blending also increase the characteristics and capabilities of fabricated objects. Moreover, discontinuous fibers as filler material also able to improve the specific properties of polymers. The improve characteristics depends on the type of filler material. Researchers are investigating in this direction due to complexity and higher cost of multi head printers. SLA is the oldest technique of 3D printing. In this process, the required object is created through the solidification of liquid resin using a photo-polymerization reaction. SLA is able to develop a mechanically stable object with a higher degree of accuracy. It can work with a wide range of engineering materials. In recent years, researchers are using SLA to develop complex 3D objects and devices of FRP composite materials for specific applications (Huang, Qin, \& Wang, 2020; Manapat, Chen, Ye, \& Advincula, 2017).

This review is focused on the recent advancement of SLA based 3D printing of FRP composites. Because, FRP composites are widely replacing traditional materials like metals, alloys, ceramics etc. in various industrial applications due to easy production and availability.

However, 3D printing of polymers is not a new research subject but FRP composites are area of interest. Moreover, some issues like the effect of fibers orientation, effect of curing time, heterogeneous composite formation and non-adhesion need to be resolved. These issues paves the way for present review article. This review paper attempts to analyze and summarize the research work carried by past researchers in the direction of SLA based 3D printing of FRP composites.

\section{STEREOLITHOGRAPHY (SLA) TECHNIQUE}

It is one of the oldest and widely used additive manufacturing technique. Various automobile industries are using this technique to print automotive components with improved surface finishes, and tight tolerances. This technique is also using in the medical field to develop 3D models of various anatomical parts by using computer scan data of the patient (Melchels, Feijen, \& Grijpma, 2010; Skoog, Goering, \& Narayan, 2014). Higher processing speed is the main advantage of this technique. Moreover, SLA is able to make strong prototypes or master patterns for the casting process.

Hull gave the concept of modern SLA method (Hull, 1984). However, before his concept, Swainson (Swainson, 1977) acquired a patent for a system, which can be used to fabricate 3D objects by using two radiation beams. These beams were intersecting in nature. In this system, beams fabricated objects through photochemically cross-linking. Moreover, Herbert (Herbert \& AJ, 1982) also developed a system using photosensitive polymers to create a 3D object using the layer-by-layer approach. Figure 1 shows the schematic of Hull's SLA system. In Hull's system, an UV radiation is used to cure photosensitive materials to form layer-by-layer 3D objects. In SLA, a physical model of the desired object is formed in layer-by-layer by using the slicing of the STL file of its 3D CAD model (Palanikumar \& Mudhukrishnan, 2020; Singh, Ramakrishna, \& Berto, 2020).

From the time of the initial concept to till date, the development of the SLA technique can be classified into a group of four generations as shown in Fig. 2. In first-generation SLA, a 3D object is formed using a laser beam for scanning liquid materials. However, this approach gained wide popularity but it had certain limitations like a lower level of efficiency. To overcome this issue, photomask technology is used in second-generation SLA and known as 
projection SLA. This technology was able to cure each level simultaneously. In third-generation SLA, a continuous liquid interface production (CLIP) approach is used to form 3D objects. The third-generation SLA technique has a higher printing speed as compared to previous techniques and reduced processing time. The fourth-generation SLA technique is used the element of intricate aperiodic 3D volumes on a time scale of seconds to create 3D objects (Huang et al., 2020; Shusteff et al., 2017; Tumbleston et al., 2015).

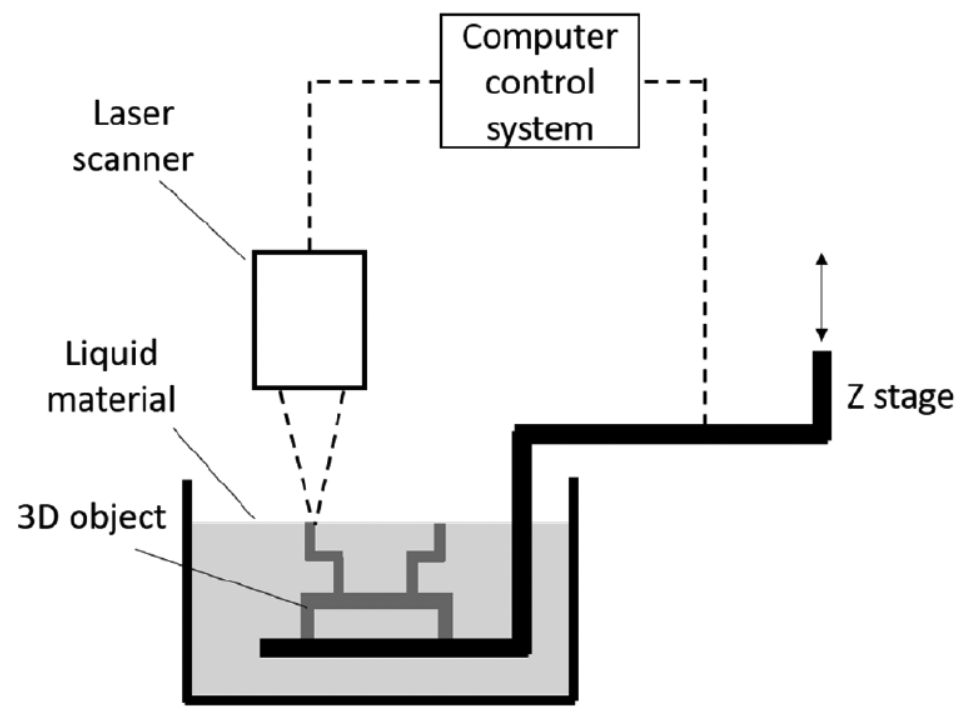

Figure 1. SLA system developed by Hull (Huang et al., 2020).

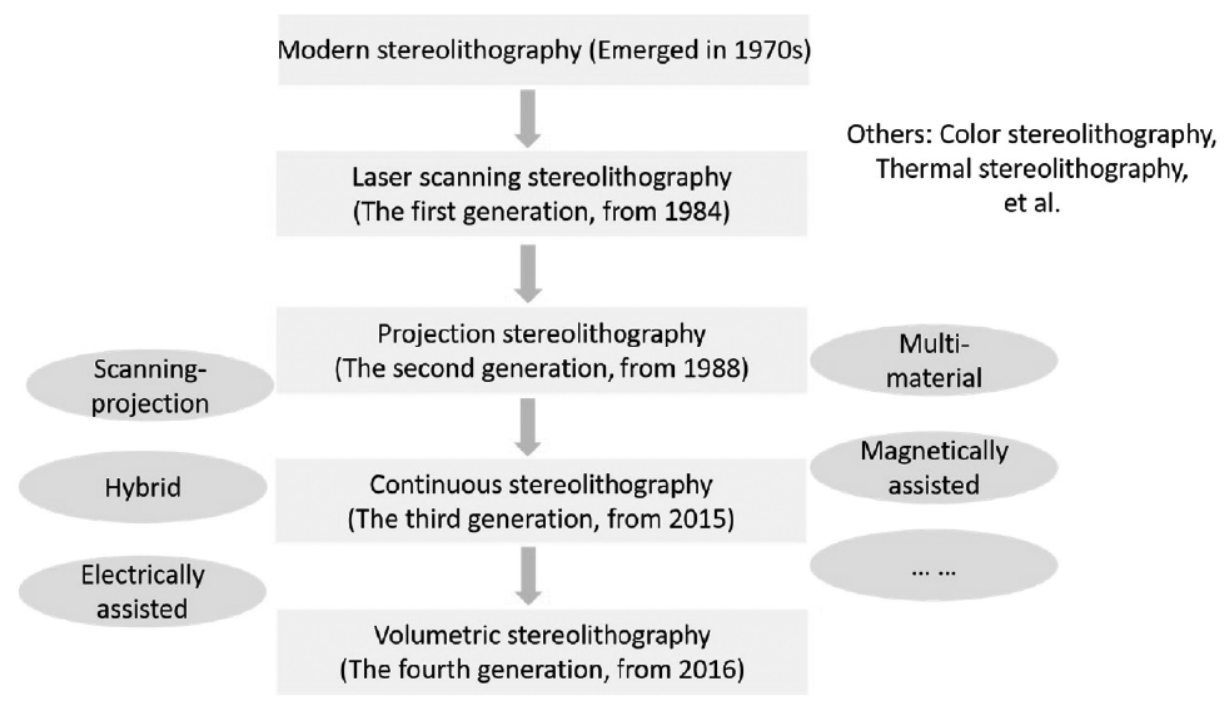

Figure 2. Development of SLA system (Huang et al., 2020). 


\section{FABRICATION OF FRP COMPOSITES USING STEREOLITHOGRAPHY}

A FRP composite materials can be fabricated by two processes through SLA method. First one is the premixing of reinforcement fiber and resin glue while another is the dispersion of the reinforcement fibers on the resin surface (Z. Lu, Lu, Cao, \& Li, 2014; Parandoush \& Lin, 2017; Popov et al., 2004). In SLA, the manual laying process is widely adopted for the fabrication of the FRP composites. Moreover, during fabrication, in most cases some additives are added to the resin to facilitate polymerization and decrease the viscosity of the resin. These additives are also able to provide a strong bonding between the reinforcement fibers and resin matrix (Goh, Yap, Agarwala, \& Yeong, 2019; Kabir, Mathur, \& Seyam, 2020; Zindani \& Kumar, 2019).

Both the nano and micro scale's continuous and discontinuous fibers can be used to fabricate FRP composites by using the SLA technique. SLA possesses a wide range of continuous and discontinuous fibers to develop FRP composites such as graphene oxide (Chiappone et al., 2017), silicate dioxide ( $\mathrm{SiO} 2$ ) (Gurr et al., 2008), titanium carbide (TiC) (Nakamoto, Kanehisa, \& Sugawa, 2013), glass (Gervasi \& Crockett, 1998), and carbon fiber mats (Karalekas, 2003) etc.

Karalekas (Karalekas, 2003) developed nonwoven glass fiber reinforced polymer composites using SLA. In this study, he showed 3D printing for single layer of glass fiber mat. He observed that multilayers of continuous fibers may be improved the mechanical properties of developed objects. Lin et al. (Lin et al., 2015) employed SLA technique to develop CNT filled polymer composites. They observed that $\% 0.2$ of graphene oxide increased the tensile strength and elongation of fabricated composite by $\% 62.2$ and $\% 12.8$ respectively.

Sano et al. (Sano, Matsuzaki, Ueda, Todoroki, \& Hirano, 2018) developed a technique to fabricate FRP composite material through SLA based additive manufacturing. In this study, he used short and continuous fibers of glass powder and fiberglass as reinforcement in a light-cured resin matrix. They observed that the mechanical properties of the formed composites in terms of tensile strength and Young's modulus of are increased up to 7.2 and 11.5 times compared to resin specimens. Figure $3(a-b)$ shows comparison in mechanical properties of the printed pure resin and glass powder and glass fiber composites.

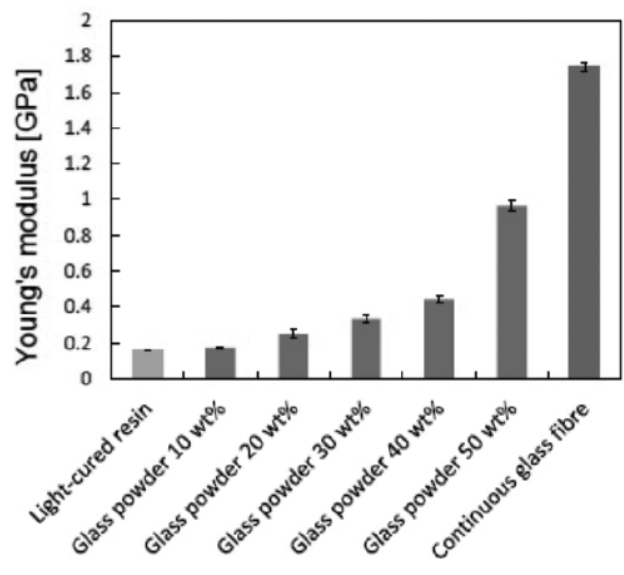

(a)

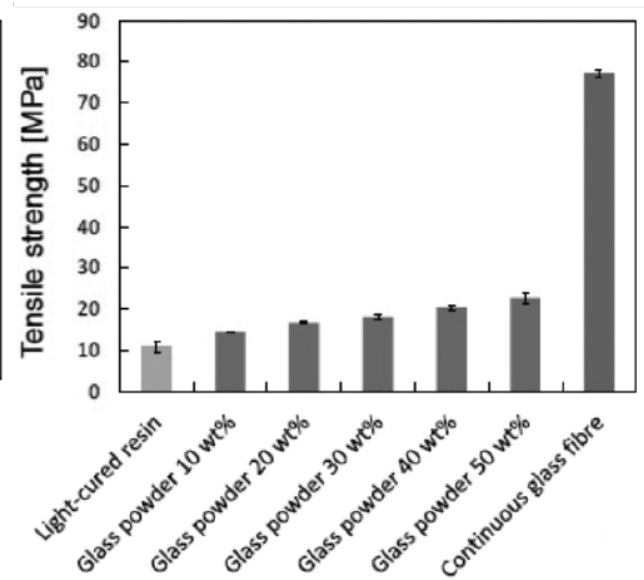

(b)

Figure 3. (a-b)Tensile strength (a) and Young's modules (b) comparison of pure resin and glass powder, and glass fiber composites with different wt \% (Sano et al., 2018). 
Yunus et al. (Yunus, Shi, Sohrabi, \& Liu, 2016) used aluminum oxide nanowires to fabricate a nanowire reinforced polymer composite. They implemented a lateral oscillation mechanism to align the reinforced fibers. They found that shear-induced alignment greatly improved the tensile strength of fabricated parts up to $28 \%$. In 3D printed parts, presence of pores and voids also decreases the mechanical properties of the created objects. So, reduction in the amount of pores or voids is essential to improve surface integrity. SLA is able to develop printed parts having low porosity (Cheah, Fuh, Nee, \& Lu, 1999; Yunus et al., 2016).

Cheah et al. (Cheah et al., 1999) added glass fibers into an acrylic-based photo-polymer and identified the effects. Based on the results, they revealed that fiber-reinforced specimens showed higher mechanical properties. They observed that elastic modulus and ultimate tensile strength are increased whereas shrinkage is reduced remarkably. In SLA technique; epoxy, polyester and photosensitive polyacrylate are some common types of employed resins (Goh et al., 2019; Nagalingam, Sundaram, Stanly, \& Retnam, 2010; Ogale \& Renault, 1991; Sakly, Kenzari, Bonina, Corbel, \& Fournée, 2014).

Lu et al. (L. Lu, Tang, Hu, \& Pan, 2018) developed a novel particle based patterning approach for 3D printing of multifunctional smart composite objects. To develop this system they used a system which is able to integrate acoustic field into the projection-based SLA system. They also verified the feasibility of the proposed approach and found it useful. Asif et al. (Asif, Chansoria, \& Shirwaiker, 2020) also used acoustic radiation forces generated by ultrasonic actuator to develop a new vat photopolymerization based 3D printing process using short crabon fibers as shown in Fig. 4. They observed that part having $1 \% \mathrm{w} / \mathrm{v}$ of fibers showed improved tensile strength due to fiber alignment. However, the addition of fiber in polymer matrix is able to improve its mechanical properties but depends on the density, size and shape of fibers.

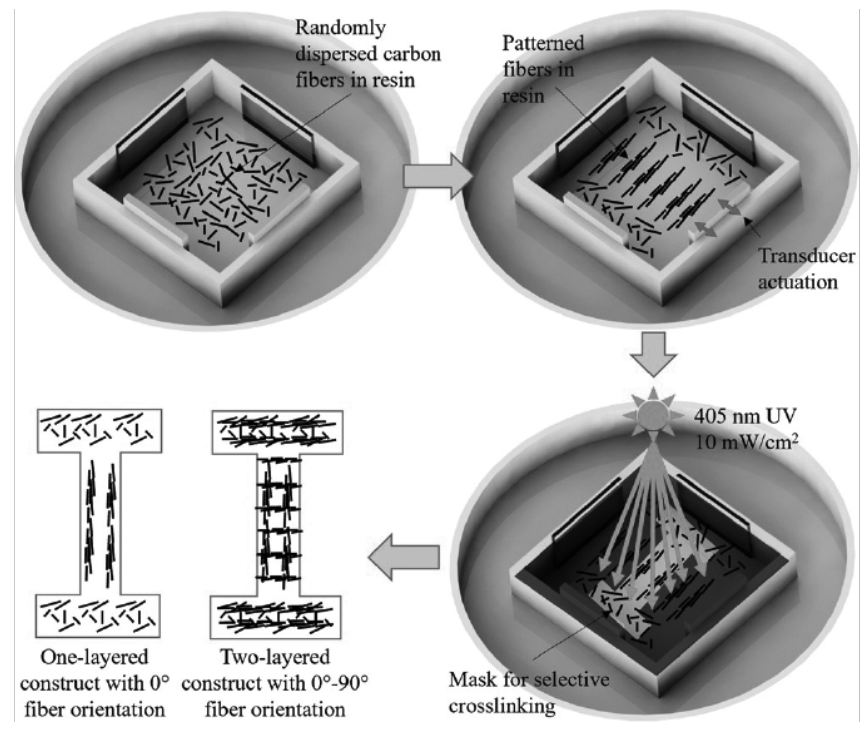

Figure 4. Alignment of fibers using ultrasonic actuation (Asif et al., 2020)

Lu et al. (L. Lu, Guo, \& Pan, 2017) developed a new SLA system for 3D printing of smart polymer composites as shown in Fig. 5. This system is used magnetic field to assist projection SLA. They developed these 3D smart polymer composites for the applications in robotics, biomedical devices, and autonomous systems. In this process, nano or micro sized ferromagnetic particles are used to deposit in liquid polymer by using a nozzle. Thereafter, a magnetic field is employed to direct the magnetic particles in to the required orientation, position and patter. Then, a digital mask image is used to cure the particles. 


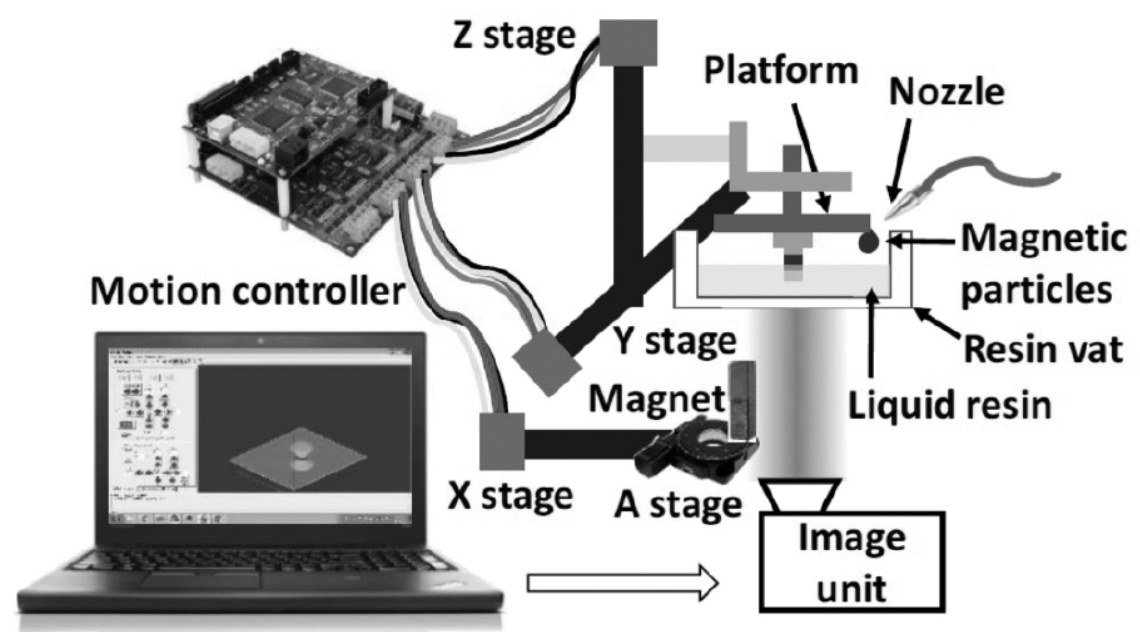

Figure 5. A schematic diagram of the M-PSL system (L. Lu et al., 2017)

During fabrication of FRP composite parts proper fiber alignment is key issue. In SLA, fibers can be aligned randomly with finer resolution. However, during SLA process, some bubbles takes place in to the formed part and reduced specific strength. Moreover, as well as the fiber are added the viscosity of resin also increased and results in sedimentation of fibers (Goh et al., 2019; Zindani \& Kumar, 2019). In SLA, fibers are align randomly and along the direction of electric and magnetic field. Fibers are also aligned according to their pattern. Scordo et al. (Scordo et al., 2019) successfully developed an electrically conductive resin for improving processing of SLA technique using the dispersion of PEDOT particles in a PEGDA matrix.

SLA approach is also using in biomedical and tissue engineering. Guillaume et al.(Guillaume et al., 2017) used SLA technique in tissue engineering. They fabricated 3D printed biodegradable composite scaffolds using photo-cross linkable poly (trimethylene carbonate) resins containing different weight percent of hydroxyapatite nanoparticles. They observed that SLA approach can be used to develop biomaterials. Kim et al. (Kim, Yeatts, Dean, \& Fisher, 2010) used SLA approach for scaffold development. They identified the effects on osteogenic signal expression and differentiation on porosity, pore size, interconnectivity, and mechanical properties on the formed scaffold. They found that SAL is able to fabricate scaffold in precise manner with higher resolution.

\section{CONCLUSION}

In this review paper, recent advancement in stereolithography based 3D printing of FRP composites are addressed. During review, it has been observed that reinforcing of short and continuous fibers can remarkably improves the mechanical properties of SLA based 3D printed parts. However, SLA process has fast printing speed but void formation during processing is a serious issue. The alignment of fibers is also one of the key issue and needed to be resolved. It has been observed that continuous fibers are able to greatly improve mechanical properties compared to short fibers. Void formation during SLA based 3D printing results in poor bonding between fibers and polymer matrix. Authors believes that SLA based 3D printing of FRP composites is a relatively new process and a lot of research is required in this field. 


\section{REFERENCES}

Asif, S., Chansoria, P., \& Shirwaiker, R. (2020). Ultrasound-assisted vat photopolymerization 3D printing of preferentially organized carbon fiber reinforced polymer composites. Journal of Manufacturing Processes, 56, 1340-1343.

Bose, S., Ke, D., Sahasrabudhe, H., \& Bandyopadhyay, A. (2018). Additive manufacturing of biomaterials. Progress in materials science, 93, 45-111.

Cheah, C., Fuh, J., Nee, A., \& Lu, L. (1999). Mechanical characteristics of fiber-filled photo-polymer used in stereolithography. Rapid Prototyping Journal.

Chiappone, A., Roppolo, I., Naretto, E., Fantino, E., Calignano, F., Sangermano, M., \& Pirri, F. (2017). Study of graphene oxide-based 3D printable composites: Effect of the in situ reduction. Composites Part B: Engineering, 124, 9-15.

Dickson, A. N., Barry, J. N., McDonnell, K. A., \& Dowling, D. P. (2017). Fabrication of continuous carbon, glass and Kevlar fibre reinforced polymer composites using additive manufacturing. Additive Manufacturing, 16, 146-152.

Frketic, J., Dickens, T., \& Ramakrishnan, S. (2017). Automated manufacturing and processing of fiber-reinforced polymer (FRP) composites: An additive review of contemporary and modern techniques for advanced materials manufacturing. Additive Manufacturing, 14, 69-86.

Gervasi, V., \& Crockett, R. (1998). Composites with gradient properties from solid freeform fabrication. Paper presented at the 1998 International Solid Freeform Fabrication Symposium.

Goh, G. D., Yap, Y. L., Agarwala, S., \& Yeong, W. Y. (2019). Recent progress in additive manufacturing of fiber reinforced polymer composite. Advanced Materials Technologies, 4(1), 1800271.

Guillaume, O., Geven, M., Sprecher, C., Stadelmann, V., Grijpma, D., Tang, T., De Bruijn, J. (2017). Surface-enrichment with hydroxyapatite nanoparticles in stereolithography-fabricated composite polymer scaffolds promotes bone repair. Acta biomaterialia, 54, 386-398.

Gurr, M., Hofmann, D., Ehm, M., Thomann, Y., Kübler, R., \& Mülhaupt, R. (2008). Acrylic nanocomposite resins for use in stereolithography and structural light modulation based rapid prototyping and rapid manufacturing technologies. Advanced Functional Materials, 18(16), 2390-2397.

Herbert, A. J., \& AJ, H. (1982). Solid object generation.

Huang, J., Qin, Q., \& Wang, J. (2020). A review of stereolithography: Processes and systems. Processes, 8(9), 1138.

Hull, C. W. (1984). Apparatus for production of three-dimensional objects by stereolithography. United States Patent, Appl., No. 638905, Filed.

Hull, C. W. (2015). The birth of 3D printing. Research-Technology Management, 58(6), 25-30.

Kabir, S. F., Mathur, K., \& Seyam, A.-F. M. (2020). A critical review on 3D printed continuous fiber-reinforced composites: History, mechanism, materials and properties. Composite Structures, 232, 111476.

Karalekas, D. (2003). Study of the mechanical properties of nonwoven fibre mat reinforced photopolymers used in rapid prototyping. Materials \& Design, 24(8), 665-670.

Kim, K., Yeatts, A., Dean, D., \& Fisher, J. P. (2010). Stereolithographic bone scaffold design parameters: osteogenic differentiation and signal expression. Tissue Engineering Part B: Reviews, 16(5), 523-539.

Kothman, I., \& Faber, N. (2016). How 3D printing technology changes the rules of the game. Journal of Manufacturing Technology Management. 
Lin, D., Jin, S., Zhang, F., Wang, C., Wang, Y., Zhou, C., \& Cheng, G. J. (2015). 3D stereolithography printing of graphene oxide reinforced complex architectures. Nanotechnology, 26(43), 434003.

Lu, L., Guo, P., \& Pan, Y. (2017). Magnetic-field-assisted projection stereolithography for three-dimensional printing of smart structures. Journal of Manufacturing Science and Engineering, 139(7).

Lu, L., Tang, X., Hu, S., \& Pan, Y. (2018). Acoustic field-assisted particle patterning for smart polymer composite fabrication in stereolithography. 3D Printing and Additive Manufacturing, 5(2), 151-159.

Lu, Z., Lu, F., Cao, J., \& Li, D. (2014). Manufacturing properties of turbine blades of carbon fiber-reinforced SiC composite based on stereolithography. Materials and Manufacturing Processes, 29(2), 201-209.

Manapat, J. Z., Chen, Q., Ye, P., \& Advincula, R. C. (2017). 3D printing of polymer nanocomposites via stereolithography. Macromolecular Materials and Engineering, 302(9), 1600553.

Melchels, F. P., Feijen, J., \& Grijpma, D. W. (2010). A review on stereolithography and its applications in biomedical engineering. Biomaterials, 31(24), 6121-6130.

Mohammadizadeh, M., Imeri, A., Fidan, I., \& Elkelany, M. (2019). 3D printed fiber reinforced polymer composites-Structural analysis. Composites Part B: Engineering, 175, 107112.

Nagalingam, R., Sundaram, S., Stanly, B., \& Retnam, J. (2010). Effect of nanoparticles on tensile, impact and fatigue properties of fibre reinforced plastics. Bulletin of Materials Science, 33(5), 525-528.

Nakamoto, T., Kanehisa, O., \& Sugawa, Y. (2013). Whisker alignment in microparts using laser stereolithography with applied electric field. Journal of Advanced Mechanical Design, Systems, and Manufacturing, 7(6), 888-902.

Noorani, R. (2017). 3D printing: technology, applications, and selection: CRC Press.

Ogale, A., \& Renault, T. (1991). 3-f) Photolithography for Composite flevelopment: Discontinuous Reinforcements. work, 5,6 .

Palanikumar, K., \& Mudhukrishnan, M. (2020). Technologies in additive manufacturing for fiber reinforced composite materials: a review. Current Opinion in Chemical Engineering, 28, 51-59.

Parandoush, P., \& Lin, D. (2017). A review on additive manufacturing of polymer-fiber composites. Composite Structures, 182, 36-53.

Popov, V., Evseev, A., Ivanov, A., Roginski, V., Volozhin, A., \& Howdle, S. (2004). Laser stereolithography and supercritical fluid processing for custom-designed implant fabrication. Journal of Materials Science: Materials in Medicine, 15(2), 123-128.

Ran, X., Mingyang, D., Yuejiao, W., Libo, G., Rong, F., \& Yang, L. (2021). Stereolithography (SLA) 3D Printing of Carbon Fiber-Graphene Oxide (CF-GO) Reinforced Polymer lattices. Nanotechnology.

Sakly, A., Kenzari, S., Bonina, D., Corbel, S., \& Fournée, V. (2014). A novel quasicrystalresin composite for stereolithography. Materials \& Design (1980-2015), 56, 280-285.

Sano, Y., Matsuzaki, R., Ueda, M., Todoroki, A., \& Hirano, Y. (2018). 3D printing of discontinuous and continuous fibre composites using stereolithography. Additive Manufacturing, 24, 521-527.

Scordo, G., Bertana, V., Scaltrito, L., Ferrero, S., Cocuzza, M., Marasso, S. L., Pirri, C. F. (2019). A novel highly electrically conductive composite resin for stereolithography. Materials Today Communications, 19, 12-17.

Shusteff, M., Browar, A. E., Kelly, B. E., Henriksson, J., Weisgraber, T. H., Panas, R. M., Spadaccini, C. M. (2017). One-step volumetric additive manufacturing of complex polymer structures. Science advances, 3(12), eaao5496. 
Singh, S., Ramakrishna, S., \& Berto, F. (2020). 3D Printing of polymer composites: A short review. Material Design \& Processing Communications, 2(2), e97.

Skoog, S. A., Goering, P. L., \& Narayan, R. J. (2014). Stereolithography in tissue engineering. Journal of Materials Science: Materials in Medicine, 25(3), 845-856.

Stavropoulos, P., \& Foteinopoulos, P. (2018). Modelling of additive manufacturing processes: a review and classification. Manufacturing Review, 5, 2.

Swainson, W. K. (1977). Method, medium and apparatus for producing three-dimensional figure product: Google Patents.

Tumbleston, J. R., Shirvanyants, D., Ermoshkin, N., Janusziewicz, R., Johnson, A. R., Kelly, D.,Ermoshkin, A. (2015). Continuous liquid interface production of 3D objects. Science, 347(6228), 1349-1352.

Wong, K. V., \& Hernandez, A. (2012). A review of additive manufacturing. International scholarly research notices, 2012.

Yan, Q., Dong, H., Su, J., Han, J., Song, B., Wei, Q., \& Shi, Y. (2018). A review of 3D printing technology for medical applications. Engineering, 4(5), 729-742.

Yavas, D., Zhang, Z., Liu, Q., \& Wu, D. (2021). Interlaminar shear behavior of continuous and short carbon fiber reinforced polymer composites fabricated by additive manufacturing. Composites Part B: Engineering, 204, 108460.

Yunus, D. E., Shi, W., Sohrabi, S., \& Liu, Y. (2016). Shear induced alignment of short nanofibers in 3D printed polymer composites. Nanotechnology, 27(49), 495302.

Zhou, L. Y., Fu, J., \& He, Y. (2020). A review of 3D printing technologies for soft polymer materials. Advanced Functional Materials, 30(28), 2000187.

Zindani, D., \& Kumar, K. (2019). An insight into additive manufacturing of fiber reinforced polymer composite. International Journal of Lightweight Materials and Manufacture, 2(4), 267-278. 